\title{
Stress analysis and deformation prediction of sheet metal workpieces based on finite element simulation
}

\author{
Ren Penghao ${ }^{1}$, Wang Aimin ${ }^{1, a}$, Wang Xiaolong ${ }^{1}$ and Zhang Yanlin ${ }^{2}$ \\ ${ }^{1}$ School of Mechanical Engineering, Beijing Institute of Technology, 100081 Beijing, China \\ ${ }^{2}$ Beijing Xin Li Machinery Co., Ltd, 100039 Beijing, China
}

\begin{abstract}
After aluminum alloy sheet metal parts machining, the residual stress release will cause a large deformation. To solve this problem, this paper takes a aluminum alloy sheet aerospace workpiece as an example, establishes the theoretical model of elastic deformation and the finite element model, and places quantitative initial stress in each element of machining area, analyses stress release simulation and deformation. Through different initial stress release simulative analysis of deformation of the workpiece, a linear relationship between initial stress and deformation is found; Through simulative analysis of coupling direction-stress release, the superposing relationship between the deformation caused by coupling direction-stress and the deformation caused by single direction stress is found. The research results provide important theoretical support for the stress threshold setting and deformation controlling of the workpieces in the production practice.
\end{abstract}

\section{Introduction}

Aluminum alloy sheet workpieces has a wide application in aerospace industry because of its light

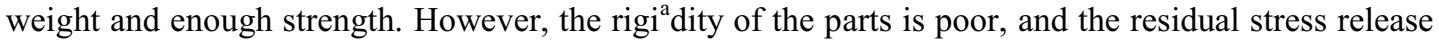
of the parts in the machining process causes deformation, which often makes the parts do not meet the accuracy requirements.

In order to solve this problem, the researchers have studied the distribution characteristics and evolution of stress after pre-stretching plate parts cutting specimen[1]; As for test and analysis of stress, they develop and improve the effective stress testing and controlling technology[2-3]; researchers also study on cutting engineering, such as a machining process and tool geometry or clamping method producing influence on distribution of residual stress and deformation of parts[46];Taking mechanism on residual stress as the starting point, the cutting force, machining stress, thermal and mechanical force are comprehensive analysed and calculated[7]. These studis on the residual stress concentrate on deformation caused by the release of stress during specific processing conditions, results of which provide important reference for the production practice, but there are also problems such as limited applicable conditions, and poor versatility. For stress conditions under other conditions, it is difficult to get a reasonable solution.

In terms of the nature of the problem, the workpiece surface and internal stress balance is broken during or after the machining process. A new equilibrium condition is established through the

\footnotetext{
${ }^{\mathrm{a}}$ Corresponding author : wangam@bit.edu.cn
} 
deformation. Therefore, it is necessary to research how stress direction and numerical value do effect on deformation of the workpiece. And on this basis, we can predict the deformation according to the stress condition of the workpiece. Then provide important theoretical support to controlling the numerical value and direction of residual stress in sensitive area by adjusting the process parameters.

\section{Theoretical model of stress and deformation of sheet metal workpieces}

According to the theory of elastic-plastic mechanics, the metal material can be regarded as an ideal elastic-plastic model, and the stress-strain behavior of metal materials is shown in Figure 1.

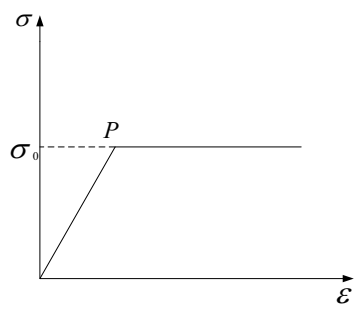

Figure 1. Stress-strain behavior of an ideal elastic-plastic model

In the figure, the stress which point $\mathrm{P}$ corresponds to is the limit yield stress $\sigma_{0}$. While the stress $\sigma<\sigma_{0}$, the material will produce elastic strain. The strain will change with the release of stress, and the workpiece, as a continuum, will produce corresponding deformation; while $\sigma>\sigma_{0}$, the material accumulates permanent strain and the deformation of the workpiece will not change even if the stress changes. Therefore, the deformation of the workpiece caused by the stress release is elastic deformation[8].

The arbitrary orientation of grain metal material is isotropic elastic material. When the machining process is completed, the residual stress will be released. The stress redistribution of the hexahedral element stress meets the following equilibrium condition:

$$
\left\{\begin{array}{l}
\frac{\partial \sigma_{x}}{\partial x}+\frac{\partial \tau_{y x}}{\partial y}+\frac{\partial \tau_{z x}}{\partial z}=0 \\
\frac{\partial \sigma_{y}}{\partial y}+\frac{\partial \tau_{z y}}{\partial z}+\frac{\partial \tau_{x y}}{\partial x}=0 \\
\frac{\partial \sigma_{z}}{\partial z}+\frac{\partial \tau_{x z}}{\partial x}+\frac{\partial \tau_{y z}}{\partial y}=0
\end{array}\right.
$$

According to the generalized Hooke's law[9], the strain caused by the stress release is :

$$
\varepsilon_{i j}=\frac{1+\mu}{E} \sigma_{i j}-\frac{\mu}{E} \sigma_{k k} \delta_{i j}
$$

where

$$
\varepsilon_{i j}=\left[\begin{array}{ccc}
\varepsilon_{x} & \varepsilon_{x y} & \varepsilon_{x z} \\
\varepsilon_{y x} & \varepsilon_{y} & \varepsilon_{y z} \\
\varepsilon_{z x} & \varepsilon_{z y} & \varepsilon_{z}
\end{array}\right]
$$




$$
\begin{gathered}
\sigma_{i j}=\left[\begin{array}{lll}
\sigma_{x} & \tau_{x y} & \tau_{x z} \\
\tau_{y x} & \sigma_{y} & \tau_{y z} \\
\tau_{z x} & \tau_{z y} & \sigma_{z}
\end{array}\right] \\
\delta_{i j}=\left[\begin{array}{lll}
1 & 0 & 0 \\
0 & 1 & 0 \\
0 & 0 & 1
\end{array}\right] \\
\sigma_{k k}=\sigma_{11}+\sigma_{22}+\sigma_{33}
\end{gathered}
$$

and $(E)$ is the elastic modulus and the $(\mu)$ is the Poisson ratio.

According to the relationship between the strain and deformation described by Lagrange displacement, the deformation of continuum can be expressed as :

$$
\varepsilon_{i j}=\frac{1}{2}\left(u_{i, j}+u_{j, i}+u_{r, i} u_{r, j}\right)
$$

The typical item expressed by engineering symbols is:

$$
\varepsilon_{x}=\frac{\partial u_{x}}{\partial x}+\frac{1}{2}\left[\left(\frac{\partial u_{x}}{\partial x}\right)^{2}+\left(\frac{\partial u_{y}}{\partial x}\right)^{2}+\left(\frac{\partial u_{z}}{\partial x}\right)^{2}\right]
$$

\section{Finite element analysis modelling}

Stress release simulation is realized by the finite element analysis, in which the ABAQUS software is used for modelling. Taking a sheet aerospace workpiece (as in Figure 2) as an example, the material is forged aluminum $2 \mathrm{~A} 12$, whose density is $2270 \mathrm{Kg} / \mathrm{m}^{3}$, elastic modulus is $71.7 \mathrm{Gpa}$, and Poisson's ratio is 0.3 . The accuracy requirement of the deformation is the edge $A B$, which is vertical to the plane on the left side of the workpiece. In order to ensure a higher simulation accuracy, and avoid the tedious operation, the workpiece is divided into 47611 hexahedron swept meshes. In order to study the influence of stress magnitude and orientation on the deformation of the workpiece, the stress condition of the workpiece is used as initial condition with quantitative input. When the process is finished, the stress is released free, so the boundary condition is not set in the simulation process.
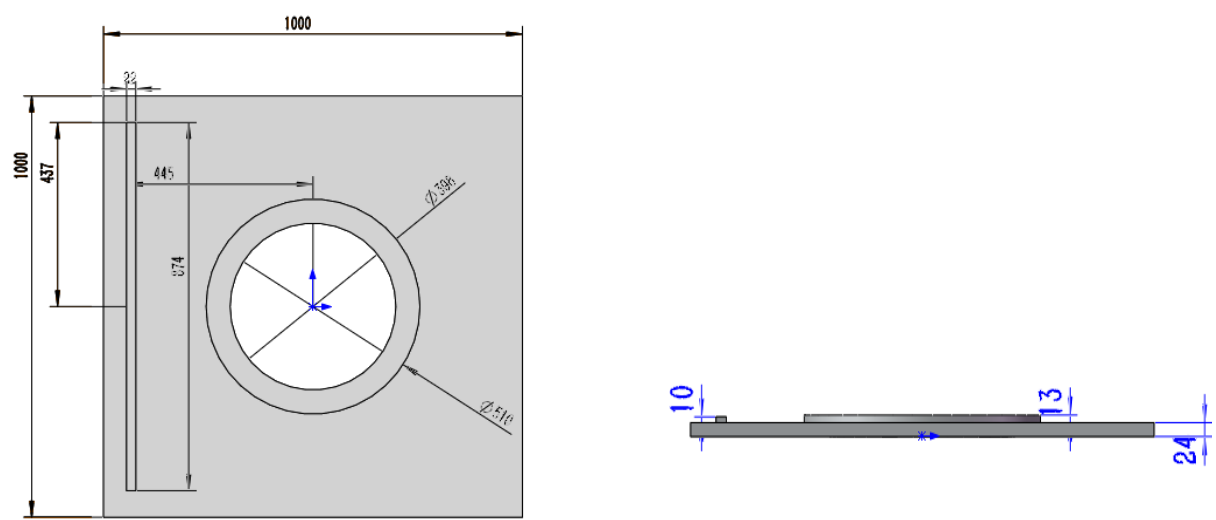

Figure 2. A sheet aerospace workpiece 


\section{Stress release simulation and deformation analysis}

\subsection{Analysis of deformation of workpiece under different stress numerical value}

The initial condition of the workpiece is shown in Figure 3. In order to explore the relationship between the stress and deformation of the workpieces, the initial stress is put into the red area in the figure manually. According to the literature and practice, the milling process will cause 100Mpa300Mpa residual stress on the surface. So place initial stress in the elements which are on the surface of the workpiece, whose numerical values are 100Mpa. The precision of the sheet is required to be perpendicular to the plane of the sheet, so after the release of the stress free (Figure.4), the deformation of the edge $\mathrm{AB}$ in the vertical direction of the plane is observed and analysed, and the deformation of each node of the $\mathrm{AB}$ side is shown in Figure 5.

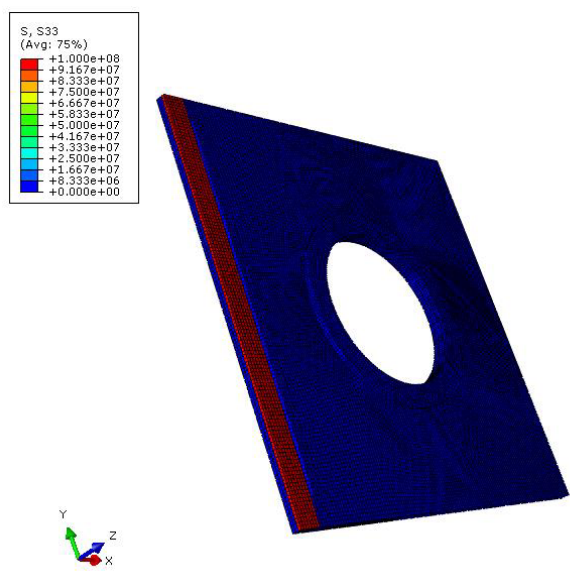

Figure 3. Initial condition of workpiece

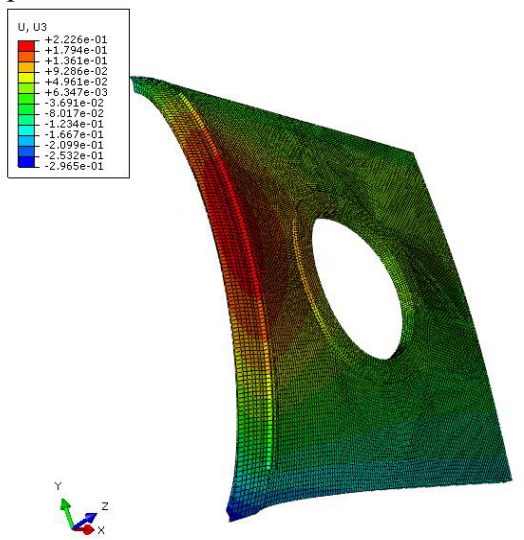

Figure 4. Workpiece after stress release free 


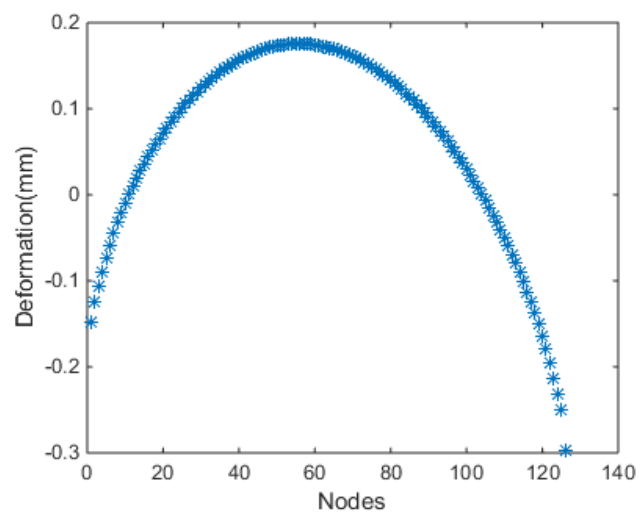

Figure 5. Deformation of edge $\mathrm{AB}$ in $\mathrm{Z}$ direction

To explore the effects of different stress numerical values on deformation of the workpiece, we do multiple analysis of different initial stress conditions and the deformation after the release of stress, the figures are similar with Figure 6 . The values of the maximum deformation are shown in table 1.

Table 1. Deformation of different stress numerical values

\begin{tabular}{cccc}
\hline \multirow{2}{*}{$\begin{array}{c}\text { Initial } \\
\text { stress/Mpa }\end{array}$} & \multicolumn{3}{c}{ The deformation of edge $\mathrm{AB} / \mathrm{mm}$} \\
\cline { 2 - 4 } & $\begin{array}{c}\text { Increasing } \\
\text { direction }\end{array}$ & $\begin{array}{c}\text { Minus } \\
\text { direction }\end{array}$ & Deflection \\
\hline 50 & 0.0877 & -0.1448 & 0.2325 \\
100 & 0.1754 & -0.2965 & 0.4719 \\
150 & 0.2639 & -0.4430 & 0.7069 \\
200 & 0.3508 & -0.5929 & 0.9437 \\
250 & 0.4371 & -0.7449 & 1.1820 \\
300 & 0.5278 & -0.8860 & 1.4138 \\
\hline
\end{tabular}

For the visual display of the maximum deformation of the workpiece under different stress numerical values, the stress-deformation figure of the workpiece is shown in Figure 6.

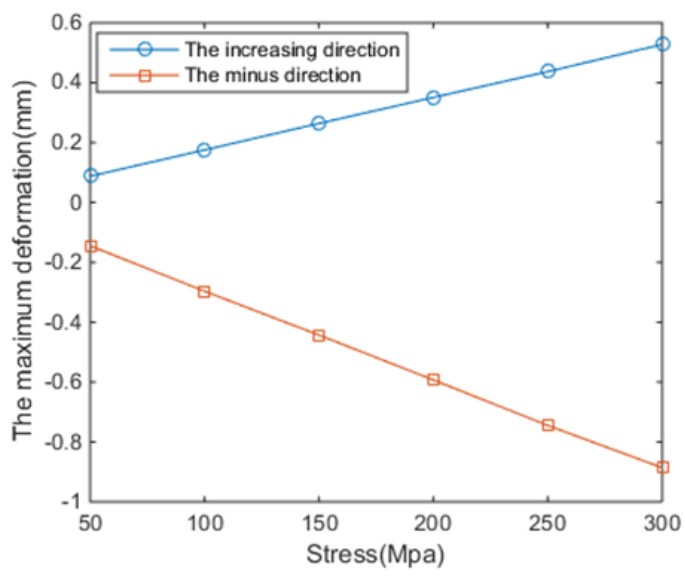

Figure 6. Deformation of edge AB

By the figure and data, it is known that the deformation of the edge of the workpiece is linearly related to the initial stress. The relationship between maximum deformation $\left(u_{\max }, \mathrm{mm}\right)$ and the stress in $\mathrm{Z}$ direction $(\sigma, \mathrm{Mpa})$ can basically fit for: 


$$
\begin{aligned}
& u_{\text {max }}^{+}=1.756 \times 10^{-3} \sigma \\
& u_{\text {max }}^{-}=-2.961 \times 10^{-3} \sigma
\end{aligned}
$$

\subsection{Analysis of deformation of parts under the condition of multi-direction stress}

According to the theory of elastic mechanics, the deformation caused by the stress release in different directions is also different. In order to explore the influence of the stress direction on the deformation of the workpiece, two more simulation experiments are carried out:

(1) Place 100Mpa initial stress in the Y direction into each element and then release free. The deformation figure is shown in Figure 7.

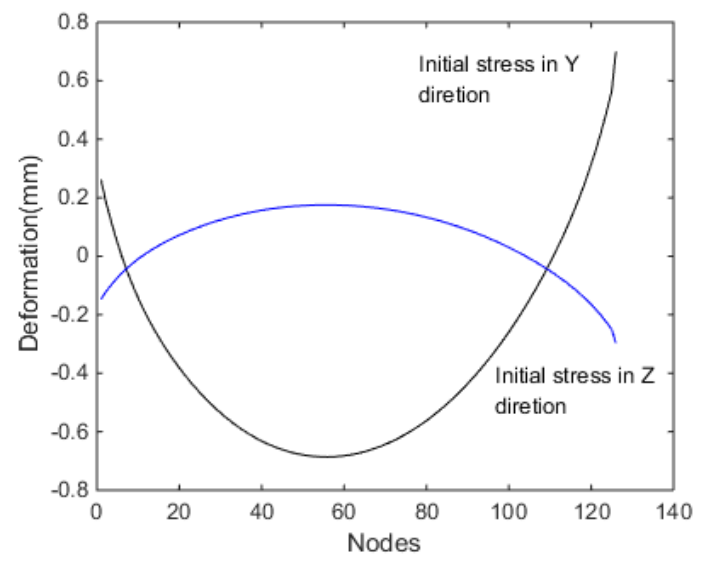

Figure 7. Deformation of edge $\mathrm{AB}$ under different stress

(2)Place 100Mpa initial stress both in the $\mathrm{Y}$ direction and $\mathrm{Z}$ direction into each element and then release free. And then observe and analyse the deformation.

In the finite element model, we select a sampling point every 15 nodes along edge $A B$, and there are 8 sampling points in total, the simulation results obtained at various that the deformation data of sampling points whose initial stress in different direction and in multi-directions are shown in Table 2. And deformation of each sampling point is plotted as curves which are shown in Figure 8.

As the table and the figure shown, deformation caused by the stress of deferent directions is deferent. After the initial stress of Y direction the release ,the workpiece bend in the minus direction, and the deformation is large; However, after the initial stress of $\mathrm{Z}$ direction release, the deformation of workpiece is in normal direction and the value is small; When the initial stress is in Y-Z coupling directions, the deformation of the edge is vector sum of which are caused by the stress in single direction.

Table 2. Deformation of each sampling point

\begin{tabular}{cccc}
\hline \multirow{2}{*}{$\begin{array}{c}\text { Sampling } \\
\text { points }\end{array}$} & \multicolumn{3}{c}{ Deformation of edge AB in Z direction } \\
\cline { 2 - 4 } & $\begin{array}{c}\text { Initial stress in } \\
\text { Y direction }\end{array}$ & $\begin{array}{c}\text { Initial stress } \\
\text { in Z direction }\end{array}$ & $\begin{array}{c}\text { Initial stress in Y-Z } \\
\text { coupling direction }\end{array}$ \\
\hline 1 & -0.2772 & 0.0363 & -0.2406 \\
2 & -0.5356 & 0.1242 & -0.4113 \\
3 & -0.6614 & 0.1669 & -0.4943 \\
4 & -0.6823 & 0.1741 & -0.5082 \\
5 & -0.6081 & 0.1488 & -0.4593 \\
6 & -0.4358 & 0.0902 & -0.3458 \\
7 & -0.1494 & -0.0073 & -0.1569 \\
8 & 0.3137 & -0.1648 & 0.1486 \\
\hline
\end{tabular}




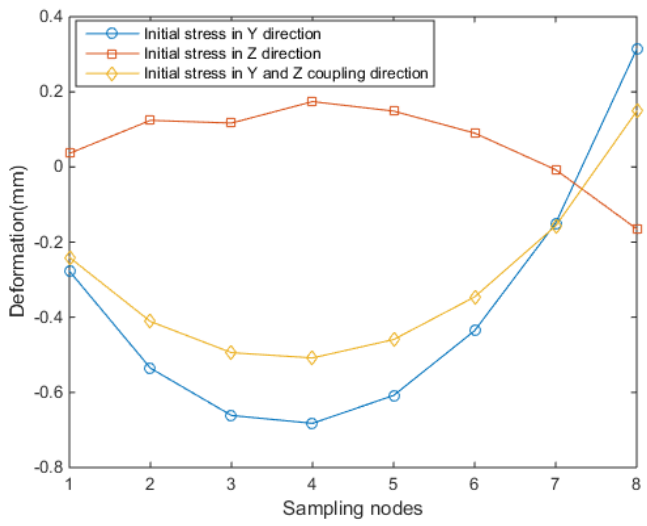

Figure 8. Deformation of sampling nodes

\section{Conclusions}

In this paper, the theoretical model of the stress and deformation of the workpiece is established based on the knowledge of elastic mechanics, and the finite element model is established by ABAQUS software. The stress release simulation experiment is carried out, and the following results are obtained:

(1) When stress is in one direction, the deformation and the numerical value of stress get into a linear relationship. The deformation increases (decreases) with initial stress increasing (decreasing). To guarantee the precision of the workpiece, we should control the stress reasonably

(2) The initial stress in different directions cause different sizes and directions of deformation of the workpiece. For sheet metal workpieces, the stress release which is parallel to the plane direction of the sheet will cause large deformation. And this kind of stress should be controlled rigorously.

(3) The deformation caused by multi-direction stress release is vector sum of which are caused by the single direction stress release. According to this, in order to guarantee the precision of the workpiece, if the milling process produce larger stress in $\mathrm{Y}$ direction, stress in $\mathrm{Z}$ direction can be applied into the workpiece to get deformation of vertical offset in the direction of the plate surface to ensure the straightness of the edge or the flatness of the plate plane.

\section{References}

1. Wu Yunxin, Liao Kai, JMT, 1, 42-46(2010)

2. Yu Wei. Analysis of machining deformation of Aeronautical thin wall parts based on residual stress (2004)

3. Wu Yunxin, Gong Hai, Liao Kai. Journal of South China University of Technology (NATURAL SCIENCE EDITION), 39, 90-94(2011)

4. Wang Zhaojun, Chen Wuyi, AAAS, 17, 175-179(2005)

5. Omar Fergani .Xiaohui Jiang . Yamin Shao . Int J Adv Manuf Technol 83, 1153-1160. (2016)

6. Li Chun, Cheng Hui Ming, Yang Feng Jun New Technology \& New Process 11, 70-73 (2012)

7. Jiang Xiaohui. The formation mechanism of residual stress and the control method of machining accuracy of complex thin wall parts. (2014)

8. Xu Zhilun. Elastic mechanics. (Higher Education Press, 2013)

9. Chen Huifa, A.F. Salipu. The elastic and plastic mechanics. (Chinese Building Industry Press, 2013) 